\title{
A 3D-Spatial-Spectral Feature Network for Hyperspectral Remote Sensing Image Classification
}

\author{
Douglas Omwenga Nyabuga ${ }^{1}$, Guohua Liu ${ }^{1+}$ and Michael Adjeisah ${ }^{1}$ \\ ${ }^{1}$ School of Computer Science and Technology, Donghua University, Shanghai City 201620, China
}

\begin{abstract}
Hyperspectral images (HSIs) are commonly applied in environmental monitoring, urban mapping, crop study, and mineral identification. These applications recurrently call for the distinguishing of the class of each pixel. Although several convolutional neural network (CNN) models have been proposed by recent researchers, none of them have been established as promising in terms of classification accuracy because of the wealth of information involved in these sorts of images for the classification of hyperspectral remote sensing images. Also, the high-dimensionality of the information, the problem of inseparability, and the limited availability of training samples are still an open challenge. This research proposes a novel convolutional neural network 3D spatial-spectral network (Model3DSN) model for the classification of hyperspectral remote sensing data, i.e., Indian Pines, Salinas Scene, and PaviaU. First, we deployed the principal component analysis (PCA) technique for low-dimensionality reduction of pixels and then 2-D and 3-D convolutions for discriminative spectral-spatial feature learning. We compared Model3DSN's efficiency against the existing spatial-spectral state-of-the-art (SOTA) models. The high accuracy achieved with the Model3DSN demonstrates its efficiency as a SOTA method for HSI remote sensing image classification, thus providing an in-depth interpretation of HSI images.
\end{abstract}

Keywords: hyperspectral, remote sensing, convolutional neural network, classification.

\section{Introduction}

Hyperspectral images (HSIs) are commonly used in various areas, including environmental monitoring, urban mapping, mineral identification, and crop analysis. This is attributed to the enormous amount of information contained in these categories of images which, by mixing rich spectral-spatial information, promote better land characterization and study [1].

Studies show that spatial features are useful in enhancing the representation of HSI data and improving the accuracy of its interpretation. As a result, it has contributed to the publication of many articles such as [2] in the field of spatial feature extraction in recent years. Supplementing spectral information with spatial information for HSI image classification has recently attracted considerable interest, which researchers have begun to combine both spatial and spectral information [3], [4] in the background of active learning (AL) in remote sensing. Generally, the inclusion of spatial information in the classification of images is directly related to the pixel neighbourhood concept. In previous years, researchers have suggested some spatialspectral-based methods. For example, Zhang et al. [5] introduced a dual-channel CNN that combines both 1D CNN and 2-D CNN to derive features. Hamida et al. [6] developed a 3-D convolutional neural network (3D-CNN) approach that allows joint spectral-spatial details. However, HSI data, together with its highdimensional, possess several elements that make the exercise of classification difficult. Often, it suffers from high intra-class variation comparable to very high-resolution (VHR) images, resulting from unregulated reflectance changes collected by the spectrometer.

In previous years, research conducted reveals some factors influencing the classification results achieved with HSI images. First, the high spectral feature dimension (i.e. hundreds of coupled spectral channels) creates the Hughes effect that can deeply decline the accuracy of classification [7]. For example, indistinguishable spectral features (e.g., identical building materials for both parking lots and roofs in the city area) [8] can be shared by different objects, making it even harder to classify HSIs using spectral details.

${ }^{+}$Corresponding author. Tel.: +8615216822311

E-mail address: ghliu@dhu.edu.cn 
Second, the accuracy of classification suffers a lot from substantially enhanced spatial resolution. In particular, rich data generated by high-resolution images leads to an increased intra-class disparity and a decrease in the inter-class disparity in both spectral and spatial domains [9], resulting in a low accuracy interpretation and inhibits the classifier's efficacy. For image classification, it is important to bring structural and contextual knowledge into the spatial domain as it is widely acknowledged. Appertaining to these two aspects of the problem, therefore, there is need to stipulate the building of effective dimension reduction (DR) and spatial feature extraction approaches [10].

PCA is an example of unsupervised feature technique for feature extraction used to derive orthogonal features from a data set and decrease the feature space's dimensionality [11]. Authors such Konstantinos et al. [12] used Randomized PCA to minimize the number of spectral channels and then combined spectral-spatial features via CNN. To this end, we suggest the use of the PCA technique to understand the intra-class variability as well as the correlation among the various classes in the spectral space. On the other hand, it improves the separability among classes, decreases, and brings a balance of the intra- and inter-class criteria in our proposed model. While PCA can effectively project spectral information into a low-dimensional representation, different artifacts which share similar spectral properties cannot be differentiated. Many DLbased techniques have lately been designed to address the stated challenges that demonstrate magnificent promise in the analysis of HSI data. Furthermore, only a few researches have mainly focused on the study of hyperspectral images using an integration of both spectral-spatial features. Therefore, for HSI remote sensing image classification, spatial characteristics are incorporated with spectral features in our novel convolutional neural network 3D spatial-spectral network (Model3DSN) proposed model. The main achievements and contributions of this study are thus defined as:

- Since neighbor intensities of the hyperspectral images are closely correlated at such a high spatial resolution, a spectral signature may cover a lot of redundant contents. As a result, we include the principal component analysis (PCA), which is commonly considered to be a good method for reducing dimensions, to reduce the set of bands and unearth discriminative details.

- In this study, we present a 3D spatial-spectral feature network that fuses a set of 2D (spatial features) and 1D (spectral features) for joint learning of the HSI hypercubes.

We investigated the performance of the Model3DSN network using three widely used HSI data sets, including the PaviaU, Salinas Scene, and Indian Pines, to assess the efficacy of our network model.

\section{Methodology}

In this part we discuss our proposed methodology. Fig. 1 shows our proposed model, i.e., novel convolutional neural network 3D spatial-spectral network (Model3DSN) that distinguishes between 2-D and 3-D convolution operations. The proposed architecture uses defined procedures to carry out the HSI image classification process. First, we apply the PCA technique to derive the sample pixels (or principal components (PCs)) of low-dimensional from HSI, and then a 2-D-CNN to derive deep features from condensed HSI with a window size of $25 \times 25$ to evaluate the label of each pixel. We concatenate 3-D-CNN into HSI processing taking advantage of the DL's potential to automatically learn spectral-spatial features. 3D-CNN performs 3-D convolution with 3-D kernels and can derive spectral and spatial features concurrently while retaining their correlations.

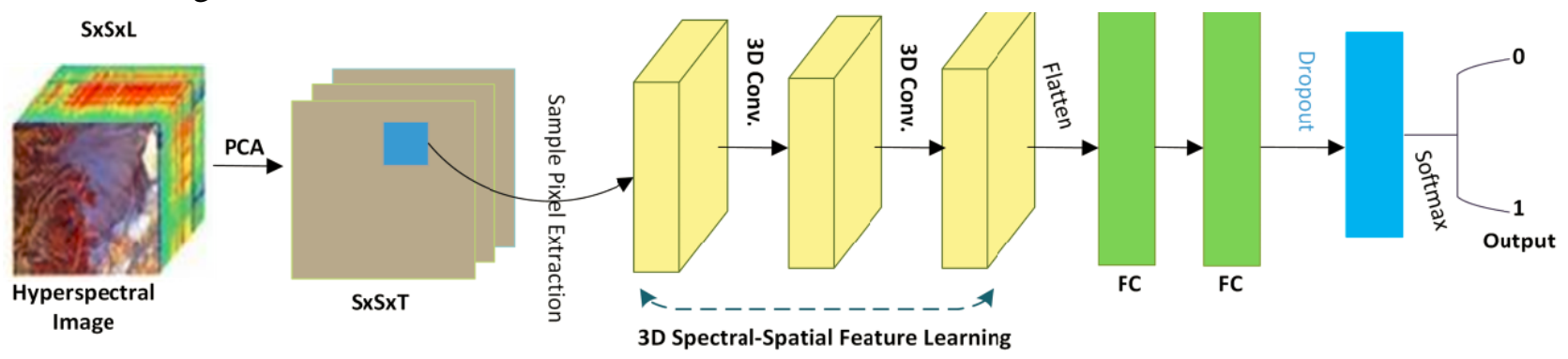

Fig. 1: The Proposed Model3DSN Framework. 
Each feature cube is considered as an independent feature in our Model3DSN model, and is thus presented as

$$
v_{l i j}^{x y z}=f\left(\sum_{h=0}^{H_{l}-1} \sum_{w=0}^{W_{l}-1} \sum_{d=0}^{D_{l}-1} k_{l j}^{h w d} v_{(l-1) i}^{(x+h)(y+w)(z+d)}+b_{l j}\right)
$$

where $D_{l}$ denotes the spectral depth of the 3-D kernel, $i$ denotes the number of feature cubes in the prior layer, $j$ denotes the number of kernels in this layer, $v_{l i j}^{x y z}$ denotes the output at position $(x, y, z)$ that is computed by convolving the ith feature cube of the previous layer with the jth kernel of the lth layer, and $k_{l j}^{h w d}$ is the $(h, w, d) t h$ value of the kernel connected to the ith feature cube in the preceding layer. As such, the output data of the lth convolution layer comprises $i \times j 3$-D feature cubes.

\subsection{Principal Component Analysis (PCA)}

Convolutional neural networks' training and prediction processes are complicated by their high dimensionality, which affects not only classification but also training and prediction complexity. PCA seeks out the best $k n$-dimensional orthogonal vectors for representing the information, where $k \leq n$. The steps discussed by [13] can be used to summarize the entire process of determining the PC and the reduced HSI data set. We can get a reduced data set from the original high-dimensional data set by following these steps, which is the primary goal for dimensionality reduction in PCA.

Given the data matrix $D$ of the size $M \times N$ where $M$ represents the various value of observations and $N$ representing the various feature values for each observation. The steps are summarized as:

1. Standardization of the values of each of the three HSI dataset: here $d_{i j}$ is the $i^{t^{t h}}$ sample value for $j^{\text {th }}$ feature of the dataset $D . \mu$ is the mean and $\sigma$ is the standard deviation of the dataset $D$. Finally, $d_{i j}^{\prime}$ is the standardized value of $d_{i j}$.

2. The covariance matrix computation, which is computed from the features of the original dataset.

Covariance between two feature vectors $X$ and $Y$ can be calculated since we have standardized the data set (i.e., the mean $\mu=0$ for each feature and the standard deviation $\sigma=1$ ).

$$
\operatorname{Cov}(X, Y)=\frac{\sum_{i=1}^{M}\left(X_{i}-\bar{X}\right)\left(Y_{i}-\bar{Y}\right)}{M}
$$

3. Computing of the Eigenvector and Eigenvalues from the covariance matrix in order to find out the $\mathrm{PC}$ through equation (3).

$$
k v=\lambda v
$$

where $v$ is the eigenvector, $\lambda$ is the diagonal matrix, and $k$ is the covariance matrix.

4. The number of top PCs to select and retain is concluded based on the cumulative variance of those components (or magnitude of their eigenvalues). From those, a matrix of vectors is formed called a feature vector and the cumulative sum is calculated with the expression,

$$
\frac{\lambda_{j}}{\sum_{j=1}^{d} \lambda_{j}}
$$

5. The transposition of the feature vector is computed in this step, where it is multiplied with the transposition of the original data set in order to obtain the reduced data set.

\subsection{Spectral-Spatial Features}

Remotely, let $\mathbf{Y} \in \mathbb{R}^{L \times m n}$ define an HSI with $L$ spectral channel/band and $m n$ pixels in the spatial domain. $\mathbf{Y}$ maybe interpreted as $m n$ feature vectors with $L$ dimensions, i.e., $\mathbf{Y}=\left[y_{1}, y_{2}, \ldots, y_{m n}\right]$. It can also be interpreted as a collection of $L$ 2-D images of size $m \times n$, i.e., $\mathbf{Y}=\left[Y_{1}, Y_{2}, \ldots, Y_{L}\right]$. Contiguous channels/bands in the visible wavelength spectrum are closely related and contain redundant information. First, the visible wavelength bands/channels are combined to produce a new image for three reasons: 1) to eliminate noisy pixels; 2) to eliminate redundant information; and 3) to extracting meaningful spatial information. The most commonly applied and easy image combination approach is finding the average, which is defined as 


$$
\mathbf{S}=\frac{\sum_{l=1}^{L_{v}} \mathbf{Y}_{l}}{L_{v}}
$$

where $\mathbf{S} \in \mathbb{R}^{m \times n}$ represents the average channel and $L_{v}$ represents the total number of channels in the visible wavelength spectrum.

At high-dimensional, the two fully connected (FC) layers can leverage more abstract spectral-spatial features that are largely robust and invariant. The FC layers transform the 3DSN HSI data set into an output vector $\left[y_{1}\right.$, 资,..., $\left.y_{L}\right]$ based on the number of land-use classes. The number of land-use classes is defined via the truth label vector $y=\left[y_{1}, y_{2}, \ldots, y_{L}\right]$. The 3 DSN loss function expression is prescribed as

$$
\text { Loss }=-\frac{1}{n_{\text {train }}} \sum_{i=1}^{n_{\text {train }}}\left[y_{i} \log \left(\hat{y}_{i}\right)+\left(1-y_{i}\right) \log \left(1-\hat{y}_{i}\right)\right]
$$

where $\hat{y}_{i}$ represents the similar predicted labels for the ith training sample, $y_{i}$ represents the true label, and $n_{\text {train }}$ represents the size of the training set. The entire network was trained in an end-to-end manner, where all the parameters were augmented by the adaptive moment estimation (Adam) optimizer.

The Adam technique [14], was adopted in our model majorly to maintain a decaying average of those past gradients centred on generating adaptive learning rates for each parameter and saving many past gradients, which makes it effective, fast convergent, and efficient when coping with the vanishing learning rate. We employed the rectified linear activation function (ReLU) principal activation function [15] in our proposed model. Also, we implemented another interesting regularization technique to alleviate overfitting that is early stopping, which saves the best accuracy at each epoch of the model while ensuring fast convergence. Additionally, we used the dropout technique with a probability of dropping node at 0.50 to also avert the model's overfitting due to insufficient training sampling set sizes. All the training and simulation were done using Python and executed in Google Colab GPU over 100 epochs with a batch size of 25 and 75 PCs.

\section{Experiments}

In this section, we discuss the data sets deployed in our experiments, the performance analysis metrics and finally, the compared SOTA methods. To assess and investigate the efficiency of the Model3DSN method we performed extensive HSI classification experiments over three real-world HSI remote sensing data sets downloaded from [16], including PaviaU (PU), Indian Pines (IN), and Salinas Scene (SS). We randomly selected $N=15 \%$ samples from each class to form the training data set (where $N$ denotes the number of training set samples). The remaining $85 \%$ sample sets were taken as the data set for testing. With randomly selected training samples, each experiment was repeated five times on average. Table 1 gives a detailed summary of the data sets used in our experiments.

Table 1: Public labeled hyperspectral remote sensing data set

\begin{tabular}{|c|l|c|c|c|c|c|c|}
\hline Hyperspectral Image & Pixels & Bands & Range $(\mu \mathrm{m})$ & GSD $(\mathrm{m})$ & Labels & Mode & Classes \\
\hline IN & 21,025 & 224 & $0.4-2.5$ & 20 & 10,249 & Aerial & 16 \\
\hline PU & 991,040 & 103 & $0.43-0.85$ & 1.3 & 50,232 & Aerial & 9 \\
\hline SS & 111,104 & 227 & $0.4-2.5$ & 3.7 & 54,129 & Aerial & 16 \\
\hline
\end{tabular}

*GSD -Ground sampled distance

We applied the overall accuracy (OA), average accuracy (AA), and Kappa coefficient (Kappa) assessment measures in this study to evaluate the efficiency of the Model3DSN model. Further, in order to adequately verify the validity of the Model3DSN method, the experimental results were compared with SOTA methods along with end-to-end deep learning-based techniques, which are both spatial-spectral-based (i.e., obtained via fusion of spatial-spectral information). Some of the methods we compared our model with are 2D-CNN (i.e., spatial-featured-based model) [12], 3D-CNN [6], M3D-CNN [17], SRNN [18], and HybridSN [19] (i.e., the spatial-spectral-featured models). 


\section{Results and discussions}

The results listed in Tables 2-4 are the classification accuracies of each class for the three hyperspectral remote sensing data sets, i.e., IN, PU, and SS, respectively. The highlighted bold values in the tables under the Model3DSN model indicate the classes on which our proposed model outperformed the other SOTA methods. This is attributable to the adoption of regularization techniques while maintaining the quick convergence of the Model3DSN model. The excellent performance obtained validates the performance of our model.

For the three data sets, we report in Table 5 the AA, OA, and Cohen's kappa performance. Experimental findings demonstrate that the Model3DSN classification approach presented in this study has superior results compared to the results obtained by other SOTA models of convolutional neural networks (CNNs). Quantitatively stated, the OA is greater than $98 \%$ on the experiments for the Model3DSN approach on the data sets tested. Thus, verifies our model as an efficient model that contributes significantly to potentially transformative improvements in the classification of the remote sensing scenes over the field of DL.

Table 2: The Indian Pines (IN) data set per class classification accuracies (\%)

\begin{tabular}{|c|c|c|c|c|c|c|c|c|}
\hline$\#$ & Name & \#Samples & 2D-CNN & 3D-CNN & $\begin{array}{c}\text { M3D- } \\
\text { CNN }\end{array}$ & SSRN & HybridSN & Proposed \\
\hline 1 & Brocoli_green_weeds_1 & 2009 & 61.14 & 70.84 & 93.52 & 96.75 & 100 & 100 \\
\hline 2 & Brocoli_green_weeds_2 & 3726 & 67.54 & 80.21 & 94.39 & 98.1 & 95.06 & 94.4 \\
\hline 3 & Fallow & 1976 & 73.74 & 77.42 & 88.9 & 98.46 & 99.58 & $\mathbf{9 9 . 8 6}$ \\
\hline 4 & Fallow_rough_plow & 1394 & 48.18 & 82.14 & 89.74 & 96.72 & 96.02 & 98.51 \\
\hline 5 & Fallow_smooth & 2678 & 78.44 & 87.72 & 91.49 & 98.17 & 98.3 & 99.76 \\
\hline 6 & Stubble & 3959 & 85.35 & 90.04 & 96.23 & 98.44 & 99.52 & 97.75 \\
\hline 7 & Celery & 3579 & 61.14 & 83.97 & 96.49 & 97.63 & 100 & 100 \\
\hline 8 & Grapes_untrained & 11271 & 86.14 & 90.12 & 96.48 & 98.78 & 100 & 100 \\
\hline 9 & Soil_vinyard_develop & 6203 & 50.42 & 80.51 & 93.1 & 97.43 & 100 & 100 \\
\hline 10 & Corn_senesced_green_weeds & 3278 & 68.93 & 79.33 & 92.81 & 97.67 & 97.71 & $\mathbf{9 9 . 5 2}$ \\
\hline 11 & Lettuce_romaine_4wk & 1068 & 77.41 & 83.03 & 93.62 & 98.23 & 99.57 & $\mathbf{9 9 . 8 6}$ \\
\hline 12 & Lettuce_romaine_5wk & 1927 & 69.03 & 81.65 & 93.65 & 97.36 & 96.83 & $\mathbf{9 9 . 6}$ \\
\hline 13 & Lettuce_romaine_6wk & 916 & 85.44 & 90.61 & 96.09 & 98.93 & 100 & 100 \\
\hline 14 & Lettuce_romaine_7wk & 1070 & 85.01 & 89.56 & 94.91 & 98.24 & 99.81 & $\mathbf{1 0 0}$ \\
\hline 15 & Vinyard_untrained & 7268 & 72.43 & 74.18 & 79.8 & 98.13 & 97.87 & $\mathbf{9 9 . 7}$ \\
\hline 16 & Vinyard_vertical_trellis & 1807 & 86.14 & 90.12 & 96.49 & 96.75 & 94.94 & 96.2 \\
\hline
\end{tabular}

Table 3: The PaviaU (PU) data set per class classification accuracies (\%)

\begin{tabular}{|c|c|c|c|c|c|c|c|c|}
\hline$\#$ & Name & \#Samples & 2D-CNN & 3D-CNN & $\begin{array}{c}\text { M3D- } \\
\text { CNN }\end{array}$ & SSRN & HybridSN & Proposed \\
\hline 1 & Asphalt & 6631 & 95.06 & 97.03 & 97.00 & 99.91 & 99.97 & 99.93 \\
\hline 2 & Meadows & 18649 & 96.09 & 95.54 & 94.79 & 99.78 & 100 & 100 \\
\hline 3 & Gravel & 2099 & 81.17 & 95.68 & 95.03 & 99.91 & 100 & 100 \\
\hline 4 & Trees & 3064 & 94.59 & 97.47 & 97.51 & 98.79 & 98.87 & $\mathbf{9 8 . 9 6}$ \\
\hline 5 & Painted metal sheets & 1345 & 96.55 & 98.63 & 98.66 & 99.81 & 99.83 & 100 \\
\hline 6 & Bare Soil & 5029 & 93.65 & 97.95 & 98.52 & 99.91 & 100 & 100 \\
\hline 7 & Bitumen & 1330 & 91.6 & 97.55 & 98.35 & 99.91 & 100 & 100 \\
\hline 8 & Self-Blocking Bricks & 3682 & 92.94 & 96.96 & 97.92 & 99.25 & 99.74 & $\mathbf{9 9 . 8 4}$ \\
\hline 9 & Shadows & 947 & 96.24 & 98.53 & 98.61 & 99.09 & 99.13 & $\mathbf{9 9 . 2 5}$ \\
\hline
\end{tabular}


Table 4: The Salinas Scene (SS) data set per class classification accuracies (\%)

\begin{tabular}{|c|c|c|c|c|c|c|c|c|}
\hline$\#$ & Name & \#Samples & 2D-CNN & 3D-CNN & $\begin{array}{c}\text { M3D- } \\
\text { CNN }\end{array}$ & SSRN & HybridSN & Proposed \\
\hline 1 & Brocoli_green_weeds_1 & 2009 & 98.85 & 95.45 & 94.78 & 99.99 & 100 & 100 \\
\hline 2 & Brocoli_green_weeds_2 & 3726 & 98.81 & 97.04 & 97.28 & 99.99 & 100 & 100 \\
\hline 3 & Fallow & 1976 & 98.48 & 96.27 & 96.71 & 99.99 & 100 & 100 \\
\hline 4 & Fallow_rough_plow & 1394 & 98.13 & 96.94 & 96.79 & 99.88 & 100 & 100 \\
\hline 5 & Fallow_smooth & 2678 & 98.05 & 96.47 & 97.00 & 99.85 & 100 & 100 \\
\hline 6 & Stubble & 3959 & 98.85 & 96.59 & 96.51 & 99.99 & 100 & 100 \\
\hline 7 & Celery & 3579 & 98.85 & 96.76 & 96.73 & 99.99 & 100 & 100 \\
\hline 8 & Grapes_untrained & 11271 & 92.47 & 86.79 & 89.91 & 99.99 & 100 & 100 \\
\hline 9 & Soil_vinyard_develop & 6203 & 98.85 & 96.85 & 96.98 & 99.99 & 100 & 100 \\
\hline 10 & Corn_senesced_green_weeds & 3278 & 97.67 & 95.40 & 94.59 & 99.89 & 99.71 & $\mathbf{1 0 0}$ \\
\hline 11 & Lettuce_romaine_4wk & 1068 & 98.58 & 95.16 & 95.33 & 99.85 & 99.98 & $\mathbf{9 9 . 9}$ \\
\hline 12 & Lettuce_romaine_5wk & 1927 & 98.85 & 96.00 & 95.78 & 99.98 & 100 & 100 \\
\hline 13 & Lettuce_romaine_6wk & 916 & 98.85 & 95.97 & 95.98 & 99.99 & 100 & 100 \\
\hline 14 & Lettuce_romaine_7wk & 1070 & 98.71 & 95.64 & 95.70 & 99.98 & 100 & 100 \\
\hline 15 & Vinyard_untrained & 7268 & 90.37 & 76.35 & 84.46 & 99.95 & 100 & 100 \\
\hline 16 & Vinyard_vertical_trellis & 1807 & 98.77 & 91.55 & 88.39 & 99.95 & 100 & 100 \\
\hline
\end{tabular}

Table 5: The classification accuracy of the proposed model in comparison with the reference baseline methods on a $15 \%$ training data set

\begin{tabular}{|c|c|c|c|c|c|c|c|c|c|}
\hline \multirow{3}{*}{ Methods } & \multicolumn{9}{|c|}{ Hyperspectral remote sensing data sets } \\
\hline & \multicolumn{3}{|c|}{ Indian Pines data set } & \multicolumn{3}{|c|}{ PaviaU data set } & \multicolumn{3}{|c|}{ Salinas Scene data set } \\
\hline & $\mathrm{OA}$ & Kappa & AA & $\mathrm{OA}$ & Kappa & AA & $\mathrm{OA}$ & Kappa & AA \\
\hline 2D-CNN & $82.43 \pm 1.8$ & $79.28 \pm 1.8$ & $75.21 \pm 4.1$ & $96.49 \pm 0.6$ & $94.89 \pm 0.9$ & $95.30 \pm 0.6$ & $96.32 \pm 1.1$ & $95.86 \pm 1.1$ & $96.88 \pm 0.7$ \\
\hline $3 \mathrm{D}-\mathrm{CNN}$ & $83.16 \pm 0.4$ & $80.34 \pm 0.6$ & $81.26 \pm 3.6$ & $96.51 \pm 0.6$ & $94.94 \pm 0.7$ & $96.54 \pm 2.6$ & $90.92 \pm 0.7$ & $93.21 \pm 1.2$ & $93.41 \pm 3.0$ \\
\hline M3D-CNN & $85.65 \pm 2.3$ & $84.50 \pm 2.2$ & $81.62 \pm 3.6$ & $96.54 \pm 1.5$ & $94.58 \pm 1.8$ & $97.78 \pm 2.1$ & $94.22 \pm 0.8$ & $93.54 \pm 0.9$ & $97.01 \pm 1.8$ \\
\hline SSRN & $98.56 \pm 0.2$ & $98.18 \pm 0.7$ & $88.55 \pm 1.0$ & $99.70 \pm 0.3$ & $99.65 \pm 0.8$ & $99.52 \pm 0.1$ & $99.78 \pm 0.2$ & $99.82 \pm 0.0$ & $99.78 \pm 0.0$ \\
\hline HybridSN & $98.58 \pm 0.1$ & $98.21 \pm 0.3$ & $98.14 \pm 0.5$ & $99.87 \pm 0.9$ & $99.83 \pm 0.6$ & $99.73 \pm 0.8$ & $99.97 \pm 0.8$ & $99.97 \pm 0.3$ & $99.97 \pm 0.5$ \\
\hline Model3DSN & $98.60 \pm 1.4$ & $98.40 \pm 0.2$ & $98.45 \pm 0.8$ & $99.90 \pm 0.4$ & $99.88 \pm 1.0$ & $99.81 \pm 1.5$ & $99.98 \pm 0.3$ & $99.98 \pm 0.6$ & $99.98 \pm 0.2$ \\
\hline
\end{tabular}

a) Experiment on Indian Pines data set: Table 5 shows a comparison of the classification accuracy results on the IN data set. We can see from the table, the SSRN, HybridSN, and Model3DSN methods have the highest performance. The performance of OA using Model3DSN was improved by $16.17 \%, 15.44 \%$, and $12.95 \%$, respectively, as compared to the 2D-CNN, 3D-CNN, and M3D-CNN methods. The HybridSN and Model3DSN methods' excellent performance on AA and kappa coefficients perfectly illustrates their steadiness and accuracy.

b) Experiment on PaviaU data set: The SSRN, HybridSN, and Model3DSN methods had the best classification accuracy on the PU data set, both of which are above $99 \%$, as can be seen in the comparison of the classification accuracy of the spectral-spatial methods in Table 5. Respectively, the Model3DSN method's classification accuracy is $3.41 \%, 3.39 \%$, and $3.36 \%$ higher than the 2DCNN, 3D-CNN, and M3D-CNN SOTA methods. HybridSN and Model3DSN have the highest average classification accuracy and kappa coefficient, indicating that the predicted classification results of different types of features were more consistent with the real object (reference object) information. Hence, its steadiness and accuracy.

c) Experiment on Salinas Scene data set: The classification accuracy of Model3DSN and HybridSN methods are superior on the SS dataset, as can be seen in Table 5. As compared to SOTA methods, 2D-CNN, 3D-CNN, and M3D-CNN methods, our proposed method improved the OA by 3.65\%, $9.05 \%$, and $5.75 \%$, respectively.

Broadly, the experimental results on three broadly used HSIs demonstrate that the Model3DSN approach outperformed other SOTA methods in terms of overall accuracy, this is illustrated in Fig. 2. 


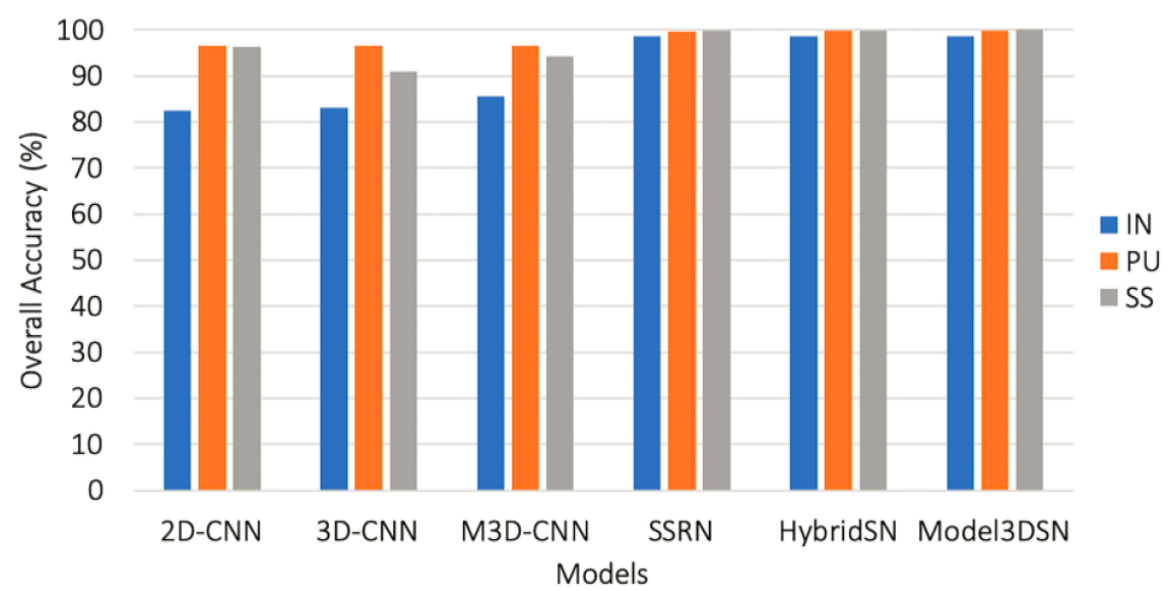

Fig. 2: The overall accuracy (OA) performance for Model3DSN proposed model compared to the state-of-the-art methods.

\section{Conclusion}

In this study, we proposed a novel convolutional neural network 3D spatial-spectral network (Model3DSN) model for the classification of hyperspectral remote sensing images. We utilized the PCA technique for the extraction of low-dimensional pixels. The 2-D and 3-D convolutional processes were adopted for spectral-spatial feature learning. Our proposed model was validated through the use of three well-known HSI data sets and compared with state-of-the-art (SOTA) methods. The Model3DSN model outperforms the SOTA method with excellent performance. Overall, our results appear consistent with all the data sets experimented. The experimental results demonstrate clear support for the spectral-spatial features in the classification of HSI images and achieving of an enhanced performance. We aim to experiment-more efficient 3-D-CNN-based HSI classification methods that can analyze unlabeled samples in future research. Unlabeled samples are much more available in HSI than labeled samples.

\section{Acknowledgements}

This work was supported in part by the Development of Shanghai Industrial Internet (Grant No. 2019GYHLW-01004).

\section{Reference}

[1] M. E. Paoletti, J. M. Haut, J. Plaza, and A. Plaza, "Deep learning classifiers for hyperspectral imaging: A review," ISPRS Journal of Photogrammetry and Remote Sensing, vol. 158. Elsevier B.V., pp. 279-317, 01-Dec-2019.

[2] L. Fang, Z. Liu, and W. Song, "Deep Hashing Neural Networks for Hyperspectral Image Feature Extraction," IEEE Geosci. Remote Sens. Lett., vol. 16, no. 9, pp. 1412-1416, Mar. 2019.

[3] E. Pasolli, F. Melgani, D. Tuia, F. Pacifici, and W. J. Emery, "SVM active learning approach for image classification using spatial information," IEEE Trans. Geosci. Remote Sens., vol. 52, no. 4, pp. 2217-2223, 2014.

[4] A. Stumpf, N. Lachiche, J. P. Malet, N. Kerle, and A. Puissant, "Active learning in the spatial domain for remote sensing image classification,” IEEE Trans. Geosci. Remote Sens., vol. 52, no. 5, pp. 2492-2507, 2014.

[5] H. Zhang, Y. Li, Y. Zhang, and Q. Shen, "Spectral-spatial classification of hyperspectral imagery using a dualchannel convolutional neural network," Remote Sens. Lett., vol. 8, no. 5, 2017.

[6] A. Ben Hamida, A. Benoit, P. Lambert, and C. Ben Amar, "3-D deep learning approach for remote sensing image classification,” IEEE Trans. Geosci. Remote Sens., vol. 56, no. 8, pp. 4420-4434, Aug. 2018.

[7] D. Donoho, "High-Dimensional Data Analysis: The Curses and Blessings of Dimensionality," AMS Math Challenges Lect., pp. 1-32, Jan. 2000.

[8] M. Fauvel, J. Chanussot, and J. A. Benediktsson, "A spatial-spectral kernel-based approach for the classification of remote-sensing images," Pattern Recognit., vol. 45, no. 1, pp. 381-392, Jan. 2012.

[9] L. Zhang, L. Zhang, D. Tao, and X. Huang, "On combining multiple features for hyperspectral remote sensing 
image classification,” IEEE Trans. Geosci. Remote Sens., vol. 50, no. 3, pp. 879-893, Mar. 2012.

[10] Plaza et al., "Spatial/Spectral Endmember Extraction by Multidimensional Morphological Operations.," IEEE Trans. Geosci. Remote Sens., 2002.

[11] M. D. Farrell and R. M. Mersereau, "On the impact of PCA dimension reduction for hyperspectral detection of difficult targets,” IEEE Geosci. Remote Sens. Lett., vol. 2, no. 2, pp. 192-195, 2005.

[12] K. Makantasis, K. Karantzalos, A. Doulamis, and N. Doulamis, "Deep supervised learning for hyperspectral data classification through convolutional neural networks," in International Geoscience and Remote Sensing Symposium (IGARSS), 2015, pp. 4959-4962.

[13] H. Abdi and L. J. Williams, "Principal component analysis," Wiley Interdiscip. Rev. Comput. Stat., vol. 2, no. 4, pp. 433-459, Jul. 2010.

[14] D. P. Kingma and J. L. Ba, "Adam: A method for stochastic optimization," in 3rd International Conference on Learning Representations, ICLR 2015 - Conference Track Proceedings, 2015.

[15] V. Nair and G. E. Hinton, "Rectified linear units improve Restricted Boltzmann machines," in ICML 2010 Proceedings, 27th International Conference on Machine Learning, 2010, pp. 807-814.

[16] "Hyperspectral Remote Sensing Scenes - Grupo de Inteligencia Computacional (GIC)." [Online]. Available: http://www.ehu.eus/ccwintco/index.php?title=Hyperspectral_Remote_Sensing_Scenes. [Accessed: 06-Jan-2020].

[17] M. He, B. Li, and H. Chen, "Multi-scale 3D deep convolutional neural network for hyperspectral image classification," in 2017 IEEE International Conference on Image Processing (ICIP), 2017, pp. 3904-3908.

[18] Z. Zhong, J. Li, Z. Luo, and M. Chapman, "Spectral-Spatial Residual Network for Hyperspectral Image Classification: A 3-D Deep Learning Framework," IEEE Trans. Geosci. Remote Sens., vol. 56, no. 2, pp. 847-858, Feb. 2018.

[19] S. K. Roy, G. Krishna, S. R. Dubey, and B. B. Chaudhuri, "HybridSN: Exploring 3D-2D CNN Feature Hierarchy for Hyperspectral Image Classification,” IEEE Geosci. Remote Sens. Lett., vol. 17, no. 2, pp. 277-281, Feb. 2019. 